\title{
Allogeneic bone marrow-derived mesenchymal stem cells attenuate hepatic ischemia-reperfusion injury by suppressing oxidative stress and inhibiting apoptosis in rats
}

\author{
GUANGXIN JIN, GONGCAI QIU, DEQUAN WU, YANHUA HU, \\ PENGFEI QIAO, CHENGJUAN FAN and FENG GAO \\ Department of General Surgery, The Second Affiliated Hospital of Harbin \\ Medical University, Harbin, Heilongjiang 150086, P.R. China
}

Received January 5, 2013; Accepted March 4, 2013

DOI: $10.3892 /$ ijmm.2013.1340

\begin{abstract}
Bone marrow-derived mesenchymal stem cells (BM-MSCs) have been shown to attenuate ischemia reperfusion (IR) injury in the heart, brain and kidney. However, their exact roles in the liver remain to be defined. Our objective was to investigate the potential effects of BM-MSCs on a hepatic IR rat model during the first $24 \mathrm{~h}$ after reperfusion, a crucial period for hepatic IR damage formation. A rat model of normothermic partial hepatic ischemia was obtained by vascular clamping for $60 \mathrm{~min}$. BM-MSCs were transplanted via portal vein injection. Injury severity, oxidative stress response and apoptosis of liver cells were assessed at 2, 6, 12 and $24 \mathrm{~h}$ after reperfusion and cell transplantation was evaluated. At 12 and $24 \mathrm{~h}$ after reperfusion, rats transplanted with BM-MSCs had significantly lower serum levels of alanine aminotransferase (ALT) and serum aspartate aminotransferase (AST), fewer damaged liver tissues, higher superoxide dismutase (SOD) and glutathione peroxidase (GSH-Px) activities and lower malondialdehyde (MDA) levels compared to rats in the sham transplantation group. At $24 \mathrm{~h}$ after reperfusion, IR rats transplanted with BM-MSCs had significantly fewer apoptotic hepatocytes, higher levels of B-cell lymphoma 2 (Bcl-2) protein, and lower levels of Bcl-2-associated X (Bax) and caspase-3 (Casp3) proteins compared to sham transplantation rats. In conclusion, BM-MSCs transplanted via the portal vein partially prevent hepatic IR injury by suppressing oxidative stress and inhibiting apoptosis during the first $24 \mathrm{~h}$ after reperfusion.
\end{abstract}

Correspondence to: Dr Dequan Wu, Department of General Surgery, The Second Affiliated Hospital of Harbin Medical University, 148 Baojian Road, Nangang, Harbin, Heilongjiang 150086, P.R. China

E-mail:wudq56@163.com

Key words: bone marrow-derived mesenchymal stem cells, hepatic ischemia reperfusion injury, oxidative stress, apoptosis, rat

\section{Introduction}

Due to the necessary clamping of the hepatic pedicle during resection of liver tumor or liver transplantation, hepatic ischemia/reperfusion (IR) injury occurs with the resumption of oxygen delivery to the liver, aggravating ischemia injury, and is considered the major cause for surgical failure (1). Reactive oxygen species (ROS) production is increased in response to IR. Ischemia is characterized by ATP depletion and oxygen free radical production $(2,3)$. Also, dysregulated electron transport chain in mitochondria may contribute to increased oxidative stress (4). However, the major source of ROS following reperfusion has been shown to be the resident macrophages in the liver, or Kupffer cells $(5,6)$. In an early phase (up to $6 \mathrm{~h}$ after reperfusion), Kupffer cells are activated, leading to increased ROS production and secretion of proinflammatory cytokines (7). In the late phase (6-24 $\mathrm{h}$ after reperfusion), recruitment of neutrophils and T-lymphocytes further increases the synthesis of ROS, signaling molecules and complement factors (8). Apoptosis is also increased in response to oxidative stress and inflammation (9). Therefore, ROS play a crucial role in the pathology of hepatic IR injury.

Mesenchymal stem cells (MSCs) are adult stem cells. Similar to other stem cells, they can renew themselves and are capable of multipotent differentiation. MSCs are considered suitable for repairing damaged organs as they are non-immunogenic and immunosuppressive cells able to differentiate into different lineages, and as they secrete a number of cytokines (10). Preventing IR damage using MSCs has been shown in the brain (11), heart (12), and kidney (13). Previous studies showed that both MSCs and MSC-conditioned medium have the potential to improve the hepatic condition in rat models of liver fibrosis or acute hepatic failure (14). These studies indicate that the hepatoprotective effects of MSCs are mainly due to their paracrine function $(15,16)$.

However, it remains unclear whether bone marrow-derived mesenchymal stem cells (BM-MSCs) could also ameliorate hepatic damage induced by IR injury. Therefore, in the present study, we generated a rat model of hepatic IR injury that closely mimics clinical conditions. Using this model, we investigated the protective role of BM-MSCs and the underlying molecular mechanisms during the first $24 \mathrm{~h}$ after reperfusion. 


\section{Materials and methods}

Animals and experimental design. Seventy-two male Wistar rats (weighing 230-250 $\mathrm{g}$ and aged 8-10 weeks) were used for this study. Prior to the experiments, the rats were housed under standard conditions at the Animal Center of the Second Affiliated Hospital, Harbin Medical University. Experimental procedures used in this study were approved by the Administrative Panel on Laboratory Animal Care of Harbin Medical University.

Rats were randomly divided into three groups of 24 rats in each group. Rats in the sham-operated group were treated by laparotomy only; rats in the other two groups were subjected to IR. Upon reperfusion, the IR-transplanted group was immediately injected with BM-MSCs via the hepatic portal vein, while the IR-control group was injected with phosphatebuffered saline (PBS) in the same manner. At 2, 6, 12 or $24 \mathrm{~h}$ after IR, $2 \mathrm{ml}$ of blood was collected from the inferior vena cava of 6 rats from each group before the animals were euthanized to harvest their livers.

Isolation of BM-MSCs. BM-MSCs were isolated using the density centrifugation method as previously described (17). Briefly, whole BM cells were flushed from the femurs and tibias of male 4-week old Wistar rats, and then fractionated in Lymphoprep ${ }^{\mathrm{TM}}$ density solution (density 1.077; Nycomed Pharma, Oslo, Norway). Following centrifugation at $800 \mathrm{x} \mathrm{g}$ for $20 \mathrm{~min}$, the cells at the interface were collected and suspended in Dulbecco's modified Eagle's medium and Ham's F-12 nutrient mixture (DMEM/F12; Gibco-Invitrogen Inc., Carlsbad, CA, USA) containing $10 \%$ fetal bovine serum (HyClone-Thermo Fisher Scientific, Waltham, MA, USA) and $1 \%$ penicillin/streptomycin. Cells were incubated at $37^{\circ} \mathrm{C}$ with $95 \%$ humidity and $5 \% \mathrm{CO}_{2}$. Forty-eight hours later, the culture medium was changed to remove non-adherent cells.

Labeling of BM-MSCs. To trace BM-MSCs following transplantation, we labeled them with the fluorescent dye PKH26 (Sigma, St. Louis, MO, USA), according to the manufacturer's instructions. Labeled cells were then cultured in growth medium for at least $24 \mathrm{~h}$ before transplantation.

Induction of hepatic IR injury and cell transplantation. Rats were anesthetized with pentobarbital sodium $(60 \mathrm{mg} / \mathrm{kg})$. A midline laparotomy was performed under aseptic conditions, the portal circulation to the left lateral and median lobes of the liver was carefully dissected, and a microaneurysm clamp was placed on the hepatic artery and portal vein to block the blood supply to these lobes. This treatment caused ischemia of $70 \%$ of the segmental liver and prevented mesenteric venous (18). The clamp was removed after $60 \mathrm{~min}$ and, immediately, $1 \times 10^{6}$ PKH26-labeled MSCs resuspended in $200 \mu 1$ PBS or PBS alone were injected into the portal vein with a 30 -gauge needle. Sham-operated rats received only the laparotomy. Surgery was closed with $4 / 0$ silk suture. When fully awake, rats had free access to food and water. During the entire procedure, the core body temperature of each rat was continuously monitored with a rectal probe and maintained at $37.0 \pm 0.4^{\circ} \mathrm{C}$ with a heating lamp.

Measurement of aspartate aminotransferase (AST) and alanine aminotransferase (ALT). To estimate the degree of hepatic IR injury, we measured the levels of serum AST and ALT. Rats were anesthetized as described above, and $2 \mathrm{ml}$ of blood was collected from the inferior vena cava with a 20 -gauge needle, placed in a microtainer tube with serum separator (Eppendorf, Hamburg, Germany), and centrifuged at $4,000 \mathrm{xg}$ for $12 \mathrm{~min}$. AST and ALT levels in the serum were measured using an automatic analyzer (Hitachi, Tokyo, Japan) and expressed as $\mathrm{U} / \mathrm{L}$.

Histological analysis. Livers were fixed in $10 \%$ buffered formalin, embedded in paraffin, and cut into $5 \mu \mathrm{m}$ sections. Sections were stained in hematoxylin and eosin (H\&E) and observed with a Nikon Eclipse 80i microscope (Nikon, Tokyo, Japan) connected to a DXM1200F digital camera. The severity of the liver injury was assessed in accordance with the modified Suzuki classification (19), modified, by a pathologist who was blinded to the experimental design. Scores for severity were: none, 0 ; minimal, 1 ; moderate, 2 ; and severe, 3 . For each rat, three liver sections were examined and three randomly selected high-power fields (x100) were analyzed in each section. The mean score for each animal was then determined by summation of all scores, divided by 9 .

Assays for superoxide dismutase (SOD), glutathione peroxidase (GSH-Px), and malondialdehyde (MDA) in livers. A portion of the injured rat liver was harvested and homogenized in ice-cold $0.9 \%$ saline. Following centrifugation at $1,500 \mathrm{x} \mathrm{g}$ for $15 \mathrm{~min}$, the supernatant was collected and used to measure the activity of SOD, GSH-Px and MDA using SOD, GSH-Px, or MDA detection kits (Nanjing Jiancheng Biotech, Nanjing, China), respectively, in accordance with the manufacturer's instructions (20).

Detection of apoptotic cells in liver tissues. We used a terminal deoxynucleotidyl transferase (TdT)-mediated dUTP nick-end labeling (TUNEL) kit (Roche Applied Science, Penzberg, Germany) to detect the apoptotic hepatocytes (21). Liver sections $(5 \mu \mathrm{m})$ were stained and six sections were analyzed for each rat. Numbers of apoptotic cells and total hepatic cells in each section were counted in three randomly selected fields (x400). An apoptosis index (AI) was expressed as the mean percentage of apoptotic cells within the total number of hepatic cells for each animal.

Western blot analysis. Whole protein extracts were prepared from liver tissues. Freshly harvested liver tissues were homogenized in a radioimmunoprecipitation assay (RIPA) lysis buffer (Solarbio Shanghai, China) and centrifuged at $12,000 \mathrm{x}$ g for $15 \mathrm{~min}$. The protein concentration in the supernatant of each sample was measured with a DCTM Protein Assay Kit (Bio-Rad, Hercules, CA, USA) according to the manufacturer's instructions. Proteins were separated using a $12 \%$ polyacrylamide gel, and then transferred to immunoblot polyvinylidene difluoride (PVDF) membranes. After blocking in 5\% milk in Tris-buffered saline containing $0.05 \%$ Tween-20 at room temperature, membranes were serially incubated with the following primary antibodies: mouse anti-Actb, rabbit anti-B cell lymphoma 2 (Bcl-2), rabbit anti-Bcl-2-associated X protein (Bax), and rabbit anti-caspase-3 (Casp3) (1:200 dilution; Santa Cruz Biotechnology, Inc., Santa Cruz, CA, USA) 

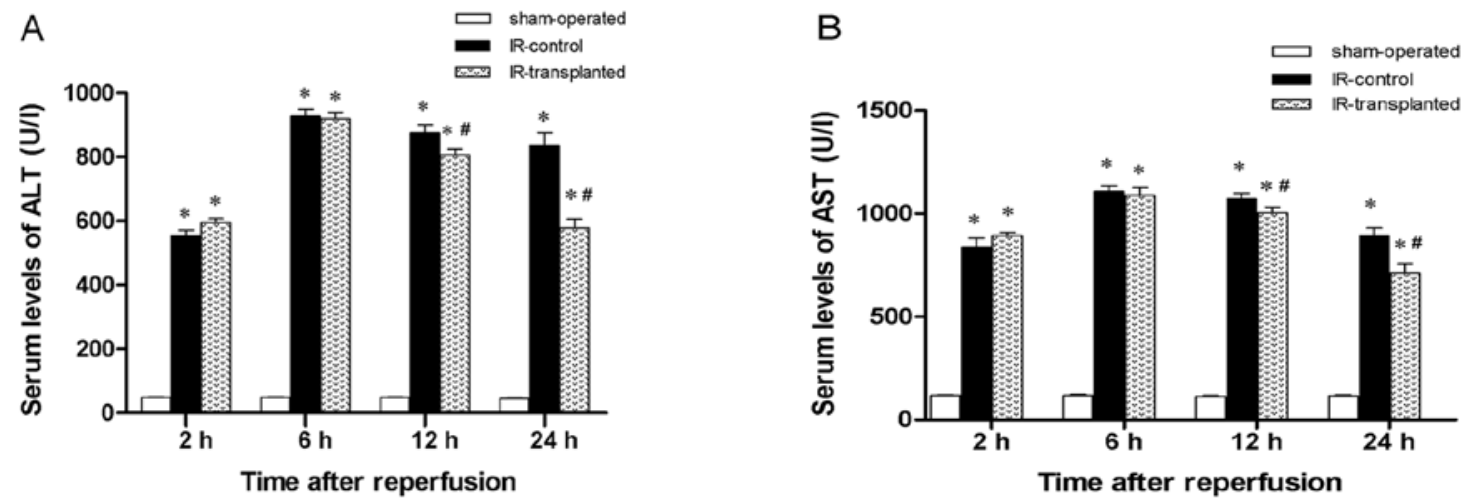

Figure 1. Effects of BM-MSC transplantation on (A) ALT and (B) AST levels in serum from rats with IR injury. Blood samples were collected at various time-points after IR and cell transplantation for ALT and AST analysis. Sham-operated rats were submitted to laparotomy only; IR-control rats were induced with IR and received PBS; IR-transplanted rats were induced with IR and received BM-MSCs. *P<0.05 IR-control or IR-transplanted vs. sham-operated rats; ${ }^{\#} \mathrm{P}<0.05$ IR-transplanted vs. IR-control rats.

at $4^{\circ} \mathrm{C}$ overnight. Membranes were then washed and incubated with fluorescence-conjugated anti-mouse or anti-rabbit IgG (1:2,000 dilution; Invitrogen). The bound secondary antibodies were analyzed with an Odyssey Infrared Imaging System (LI-COR Biosciences, Lincoln, NE, USA), normalized to $\beta$-actin.

Statistical analysis. SPSS 13.0 (SPSS Inc., Chicago, IL, USA) was used for statistical analyses. For multiple comparisons, data were analyzed using analysis of variance (ANOVA). Analysis between two groups was performed using the unpaired Student's t-test (two-tailed) where ANOVA indicated significance for the multiple comparisons. Data are reported as mean \pm standard deviation. A P-value $<0.05$ was considered to indicate a statistically significant difference.

\section{Results}

Effect of transplanted BM-MSCs on ALT and AST serum levels. To determine the degree of IR-induced hepatic injury in the rat livers receiving BM-MSCs or PBS, we measured ALT and AST levels in sera collected at 2, 6, 12 and $24 \mathrm{~h}$ after IR induction and cell transplantation. Compared to the sham-operated rats, ALT and AST levels in IR model rats were higher at every time-point, with the greatest difference $6 \mathrm{~h}$ after induction (Fig. 1). This suggests that IR injury was successfully induced in the IR model rats. At 12 and $24 \mathrm{~h}$ after reperfusion, ALT and AST levels in the IR-transplanted group significantly decreased compared to the IR-control group. This suggests that the transplantation of BM-MSCs had a protective effect against hepatic injury induced by the IR injury.

Transplanted BM-MSCs improve the histopathology of $I R$-induced livers. To further confirm the protective role of BM-MSCs in IR-induced hepatic injuries in rats, we examined the histopathology of livers harvested 2, 6, 12 or $24 \mathrm{~h}$ after IR induction and cell transplantation. At every time-point, all IR-induced livers showed sinusoidal congestion, cytoplasmic vacuolization, and focal necrosis, which are indicative of severe damage. When comparing the Suzuki scores between the livers of the IR-transplanted group and the IR-control group, improved histopathology and significantly lower Suzuki scores were found in the IR-transplanted group only at the 24 h time-point (Fig. 2).

Dynamic distribution of transplanted BM-MSCs in IR-induced livers. We therefore investigated the distribution pattern of BM-MSCs transplanted via the portal vein as a function of time. BM-MSCs were stained by PKH26 fluorescent dye in vitro. We observed that at $2 \mathrm{~h}$, transplanted PKH26-labeled cells were clustered around the main branches of the portal triad; at $6 \mathrm{~h}$, these cells had moved to the periportal area, and at 12 and $24 \mathrm{~h}$ they were scattered within the portal tract areas (Fig. 3).

Transplanted BM-MSCs attenuate the oxidative stress response in $I R$-induced livers. Previous studies have shown that the oxidative stress response is involved in IR-induced liver injury. We therefore assessed if transplanted BM-MSCs would attenuate the oxidative stress response in liver IR injury, by comparing SOD and GSH-Px activity levels, and MDA levels. We observed that SOD and GSH-Px levels were markedly decreased and that MDA levels markedly increased at 2, 6, 12 and $24 \mathrm{~h}$ in IR model rats compared with the shamoperated rats. This suggests that oxidative stress was indeed involved in IR-induced liver injury. Furthermore, we observed that transplantation of BM-MSCs significantly increased SOD and GSH-Px levels and significantly decreased MDA levels at 12 and $24 \mathrm{~h}$, compared to rats in the IR-control group (Fig. 4). These results indicate that transplanted BM-MSCs attenuated the oxidative stress response in IR-induced liver injury.

Transplanted BM-MSCs inhibit apoptosis in IR-induced livers. Increased oxidative stress in a tissue often results in apoptosis. We therefore examined apoptotic activity in the IR-model rat livers and if the transplantation of BM-MSCs protected cells from apoptosis. We first assessed apoptotic cells in the livers using TUNEL staining, and then analyzed the expression levels of the anti-apoptotic protein, Bcl-2, and the pro-apoptotic proteins, Bax and Casp3. Twenty-four hours after reperfusion and cell transplantation, the livers from the IR-model rats had a markedly higher apoptotic index 

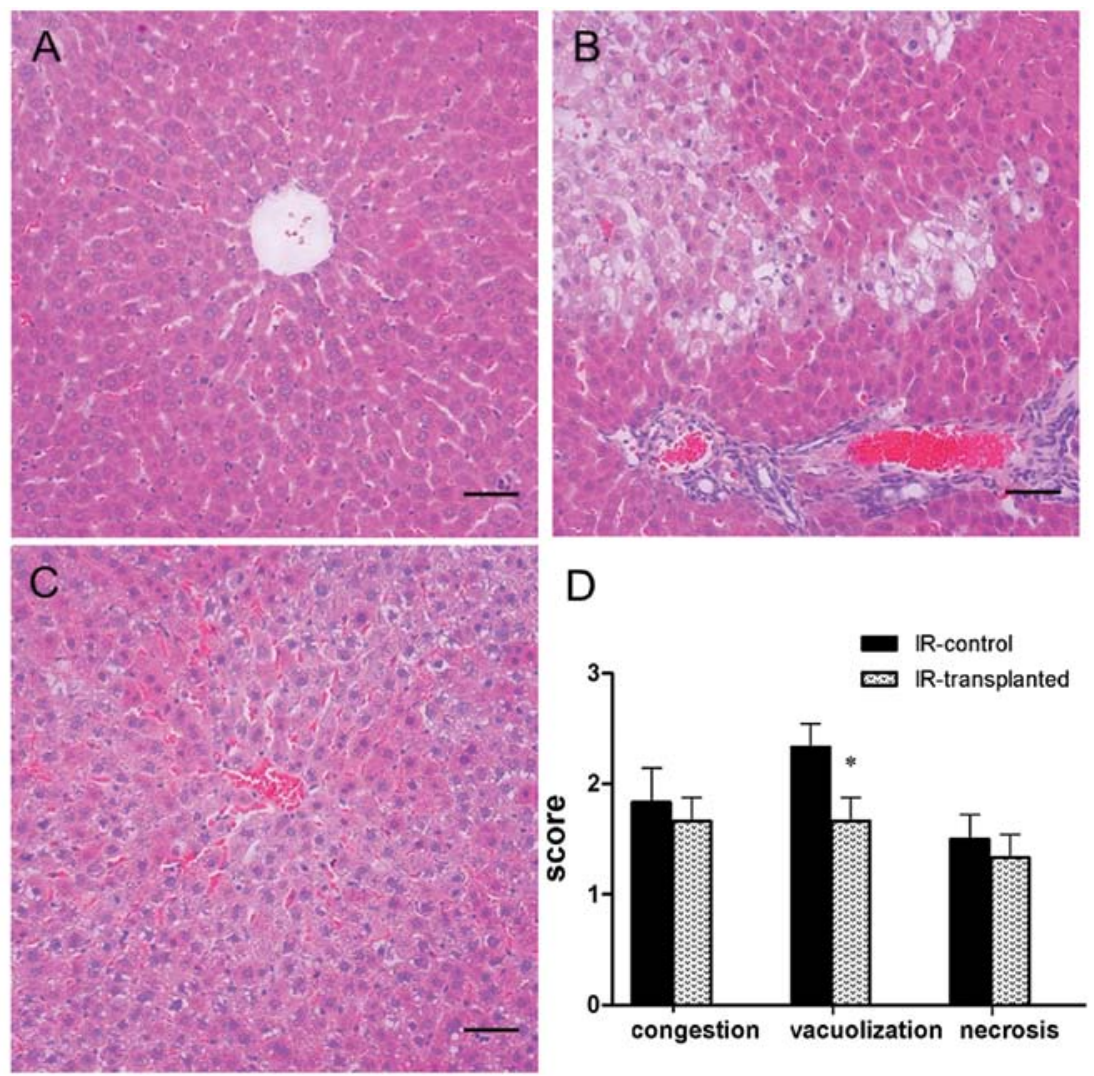

Figure 2. Histopathological analysis of livers with IR and BM-MSC transplantation. Liver tissues were harvested $24 \mathrm{~h}$ after rats were submitted to hepatic IR and cell transplantation. Liver tissue sections were stained with H\&E and scored according to the Suzuki Score System. Representative micrographs of livers from (A) sham-operated, (B) IR-control and (C) IR-transplanted rats. (D) Suzuki scores from each group. Sham-operated rats were submitted to laparotomy only; IR-control rats were induced with IR and received PBS; IR-transplanted rats were induced with IR and received BM-MSCs. ${ }^{*}<0.05$ IR-transplanted vs. IR-control rats. Scale bar, $100 \mu \mathrm{m}$.
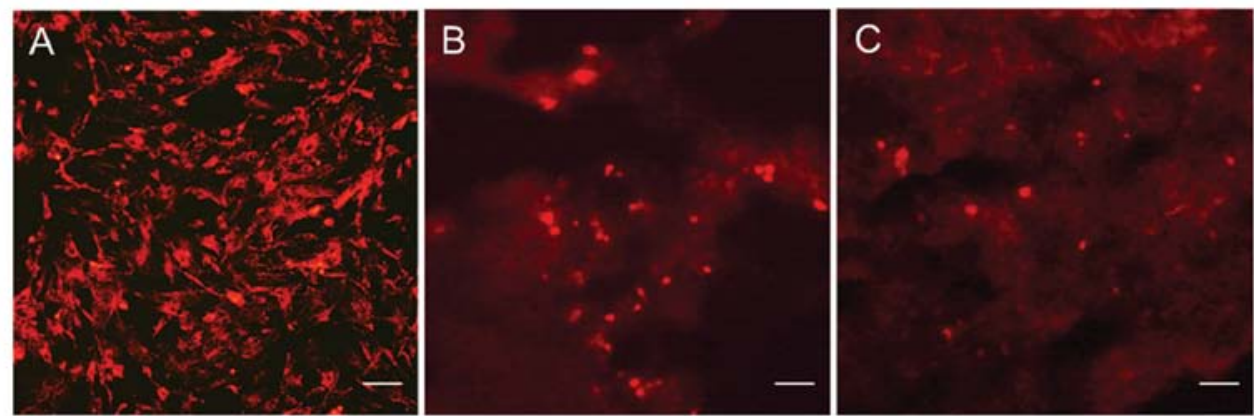

Figure 3. Dynamic localization of BM-MSCs in livers induced with IR and transplanted with PKH26-labeled BM-MSCs. Freshly stained BM-MSCs were collected and transplanted into IR-damaged livers via the portal vein. At 2, 6, 12 and $24 \mathrm{~h}$ after IR induction and cell transplantation, livers were harvested, sectioned, and examined with fluorescent microscopy. (A) PKH26-stained BM-MSCs. (B) Representative image of a liver section at $2 \mathrm{~h}$. PKH26-labeled MSCs were clustered around the main branches of the portal triad and periportal areas. (C) Representative image of a liver section at $24 \mathrm{~h}$. PKH26-labeled cells were found scattered in the portal tract area. Images at 6 or $12 \mathrm{~h}$ are not shown as no difference was observed in images from 6,12 or $24 \mathrm{~h}$. Scale bar, (A) $25 \mu \mathrm{m}$; (B and C) $100 \mu \mathrm{m}$.

than those from the sham-operated rats, suggesting that IR induction caused hepatic apoptosis (Fig. 5). This was further confirmed by a significant decrease in Bcl-2 levels, and by significant increases in Bax and Casp3 levels in IR-injured livers (Fig. 6). The apoptotic index was lower in livers from the IR-transplanted rats (Fig. 5), Bax and Casp3 levels were lower, and Bcl-2 levels were higher. Collectively, these results indicate that the transplanted BM-MSCs had a protective effect against apoptosis in the IR-induced livers.

\section{Discussion}

In the present study, we investigated the damage and molecular mechanisms of liver IR injury in a rat model as well as the protective role of BM-MSCs during the crucial first 24-h period. We found evidence of IR injury during the first $24 \mathrm{~h}$ after reperfusion. From $2 \mathrm{~h}$ after reperfusion, AST and ALT serum levels were higher than in the sham-operated rats and severe damage was observed in the histopathology. In addition, 

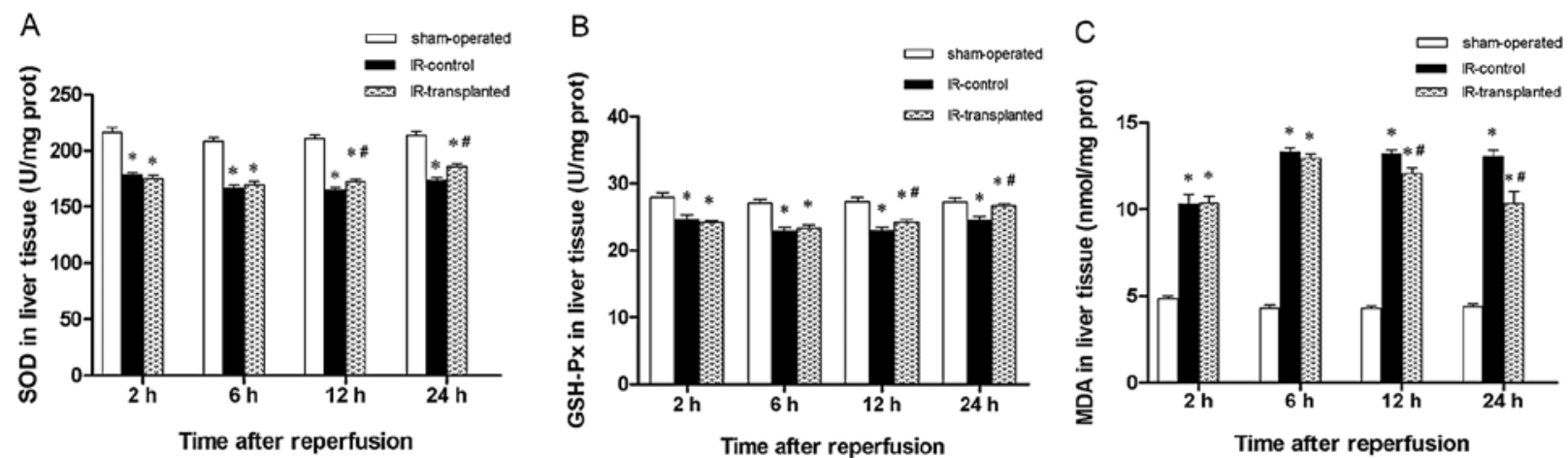

Figure 4. Effect of BM-MSC transplantation on oxidative stress response in livers induced with IR. Livers were harvested at 2, 6, 12 and $24 \mathrm{~h}$ after IR induction and cell transplantation, and the expression of (A) SOD, (B) GSH-Px and (C) MDA were assessed. Sham-operated rats were submitted to laparotomy only; IR-control rats were induced with IR and received PBS; IR-transplanted rats were induced with IR and received BM-MSCs. "P $<0.05$ IR-control or IR-transplanted vs. sham-operated rats; ${ }^{*} \mathrm{P}<0.05$ IR-transplanted vs. IR-control rats.
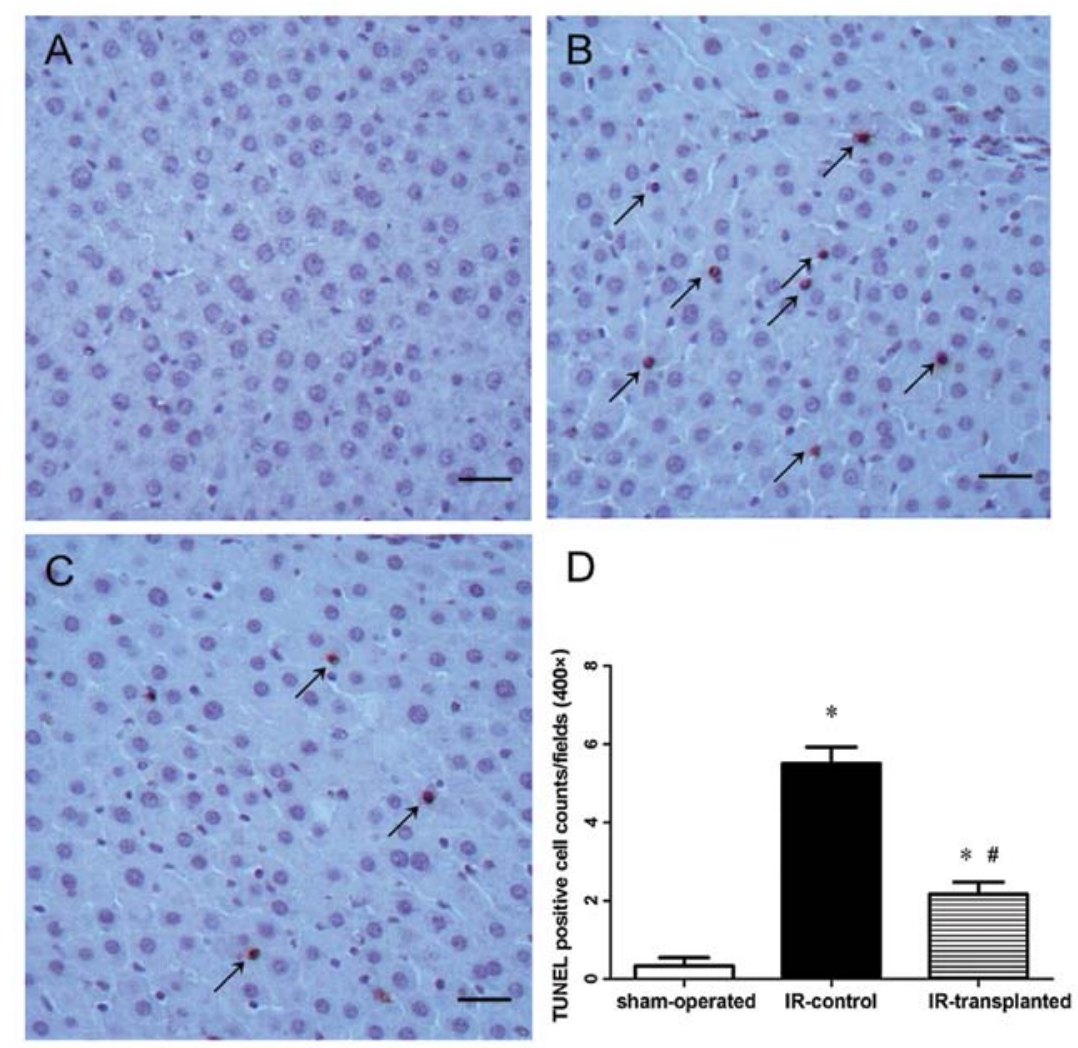

Figure 5. BM-MSC transplantation protected livers against IR injury. Livers were harvested and sectioned for TUNEL staining $24 \mathrm{~h}$ after IR induction and cell transplantation. Representative images from the (A) sham-operated group, (B) IR-control group and (C) IR-transplanted group are presented. Arrows point to TUNEL-positive apoptotic cells. (D) Apoptotic scores from the livers in each group. Sham-operated rats were submitted to laparotomy only; IR-control rats were induced with IR and received PBS; IR-transplanted rats were induced with IR and received BM-MSCs. "P<0.05 IR-control or IR-transplanted vs. sham-operated rats; ${ }^{*} \mathrm{P}<0.05$ IR-transplanted vs. IR-control rats. Scale bar, $200 \mu \mathrm{m}$.

evidence of increased oxidative response was observed from $2 \mathrm{~h}$ after reperfusion, while apparent apoptosis was observed at $24 \mathrm{~h}$. We also observed that BM-MSCs transplanted via the portal vein exerted a protective effect against hepatic IR damage during the late phase following reperfusion. This observation was further supported by the dynamic distribution of BM-MSCs; $24 \mathrm{~h}$ after reperfusion, the majority of the cells were scattered in the portal tract area of the livers.

For a therapeutic use of stem cells to be efficient, cells must be easy to isolate, available in large amounts, able to expand in vitro, and able to survive in vivo in sometimes harsh conditions. Based on these criteria, BM-MSCs are suitable candidates (22). Once injected, they are recruited at the site of injury via homing mechanisms involving the CXCR4 receptor and the stromal derived factor-1 (SDF-1) (23). Once implanted, stem cells improve the injured tissue by secreting growth factors [including vascular endothelial growth factor (VEGF), hepatocyte growth factor (HGF), and insulin-like growth factor 1 (IGF-1)] $(24,25)$, by differentiating into organ cells, by transdifferentiating the organ cells and by inducing 


\section{A}

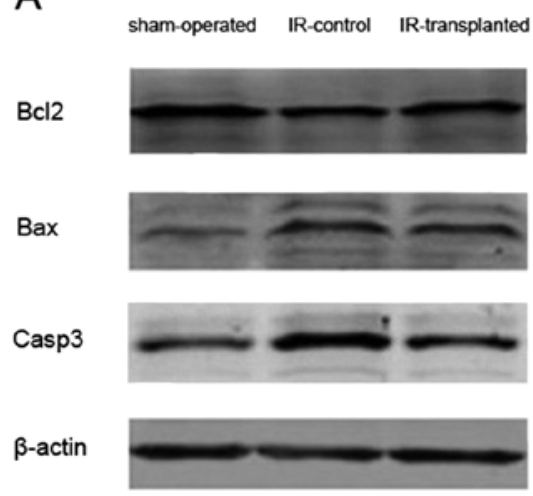

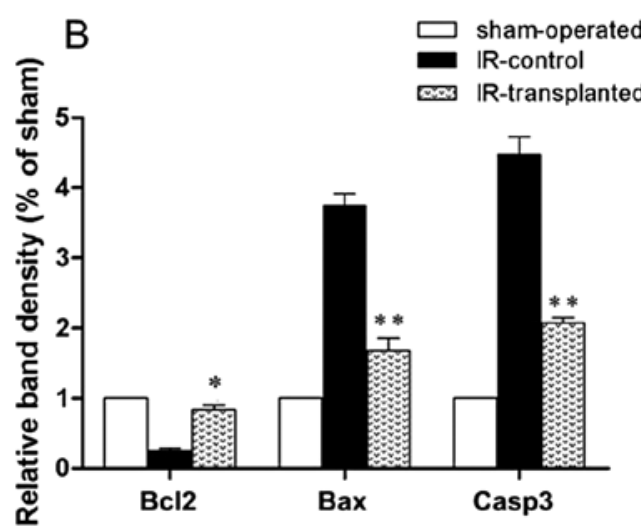

Figure 6. Analysis of anti- and pro-apoptotic proteins in livers with IR injury and receiving BM-MSC transplantation. Livers were harvested 24 h after IR induction and cell transplantation to prepare protein extracts. (A) The anti-apoptotic protein, Bcl-2, and the pro-apoptotic proteins, Bax and Casp3, were analyzed by western blotting. (B) Relative levels of these proteins in relation to $\beta$-actin in each sample presented in (A). Sham-operated rats were submitted to laparotomy only; IR-control rats were induced with IR and received PBS; IR-transplanted rats were induced with IR and received BM-MSCs. "P<0.05 IR-transplanted vs. IR-control rats. ${ }^{* *} \mathrm{P}<0.01$ IR-transplanted vs. IR-control rats.

neovascularization (11-13). Results from the present study are in agreement with results obtained from studies of other organs, such as the heart (12), brain (11) and kidney (13). Our results show that BM-MSCs homed to IR-injured liver tissue and improved liver conditions, as assessed by apoptosis measurement. Also, the protective role of BM-MSCs was observed during the late phase of the crucial period (from 12 to $24 \mathrm{~h}$ after reperfusion) of IR damage induction, indicating that BM-MSCs were viable and able to function in a short time after transplantation. Differentiating into hepatocytes or hepatocyte-like cells requires time; therefore, it is more likely that the short-term effects of transplanted BM-MSCs on IR injury were achieved via a paracrine mechanism, rather than via repopulation.

The oxidative stress response has long been recognized as central to the pathogenesis of hepatic IR injury (26). In the present study, we observed that at 2, 6, 12 and $24 \mathrm{~h}$ after reperfusion, the levels of the antioxidative enzymes SOD and GSH-Px were significantly decreased, while MDA levels, a marker for oxidative activity, was significantly increased, compared with the sham-operated control rats. Therefore, our observations are consistent with other studies showing that the oxidative stress response is involved in the formation of hepatic IR injury in the first $24 \mathrm{~h}$.

We demonstrated in the present study that BM-MSCs transplanted via the portal vein attenuated the oxidative stress response in IR-injured livers. The suppressive role of BM-MSCs in the oxidative stress response was also observed in other studies of drug-induced animal models of liver disease. In a mouse model of liver cirrhosis induced by carbon tetrachloride $\left(\mathrm{CCl}_{4}\right)$, transplantation of human BM-MSCs suppressed the oxidative stress response and improved liver conditions (27). In a rat model of hepatic injury induced by $\mathrm{CCl}_{4}$, administration of BM-MSCs induced a cytoprotective response by an antioxidative process (28). MSC suppression of the oxidative stress response was also shown in rats with IR-induced lung and kidney injury (29). Our study showed that at 12 and $24 \mathrm{~h}$ after reperfusion, the BM-MSC transplants attenuated the decrease in SOD and GSH-Px, and the increase in MDA, that otherwise occurred in the IR-control rats that received PBS only. These results indicate that BM-MSCs are capable of reducing the oxidative stress response in livers subjected to IR injury.

Apoptosis plays an important role in IR-injured liver and is triggered either by the mitochondria or by tumor necrosis factor (TNF) signaling. Indeed, hypoxia leads to reduced ATP production and ATP-dependent cellular events are stopped (30). Mitochondria also regulate apoptosis by caspase activation (31). Hypoxia-induced injury also induces an immune response resulting in the secretion of cytokines (including TNF, SDF-1 and interleukins) and the attraction of a number of cells, including MSCs. TNF signaling activates caspases, resulting in apoptosis (32). During reperfusion, immune and endothelial cells are activated and produce ROS, which directly damage nearby cells and activate Ras, increasing apoptosis (33). In a mouse model of IR-induced hepatic injury, administrating the antioxidant mangafodipir reestablished oxidative balance and suppressed apoptosis (34). Using the same mouse model, Yu et al (35) demonstrated that the Notch-Janus kinase 2 (JAK2)/signal transducer and activator of transcription 3 (STAT3) signaling pathway protected hepatocytes from IR injury by attenuating oxidative stress and inhibiting apoptosis in vitro and in vivo. In the present study, we observed apoptosis in liver tissues subjected to IR $24 \mathrm{~h}$ after reperfusion, as shown by an increased apoptotic index, decreased expression of Bcl-2, and increased expression of Bax and Casp3. Furthermore, we showed that transplantation of BM-MSCs via the portal vein decreased apoptosis. Therefore, our results are consistent with previous studies suggesting that apoptosis is involved in IR-induced hepatic injury and that BM-MSCs inhibit apoptosis.

BM-MSCs inhibit hepatocyte apoptosis by secreting cytokines, thus regulating cellular signal transduction pathways. In rats, BM-MSCs secrete VEGF, which attenuates myocardial IR injury by activating the PI3K signaling pathway (36), and the PI3K pathway can regulate the expression of Bcl-2, an antiapoptotic protein (37). In rat neurons, MSCs secrete cytokines that reduce chronic ethanol-induced injury by modulating the extracellular-signal-regulated kinase (ERK)1/2 pathway (38). The ERK1/2 pathway regulates apoptosis by increasing the 
Bax/Bcl-2 ratio, Casp3 levels and TNF levels (39). In the present study, we observed, at $24 \mathrm{~h}$ after reperfusion and BM-MSC transplantation, that Bcl-2 levels, an anti-apoptotic protein, was increased, and that the expression levels of pro-apoptotic proteins, Bax and Casp3, were decreased. We thus inferred that BM-MSCs inhibit apoptosis in IR-induced hepatic injury via their paracrine activity.

In conclusion, we demonstrated that in a rat model of IR injury, hepatic injury occurred within $24 \mathrm{~h}$ of reperfusion and that the oxidative stress response and subsequent apoptosis were involved in the process of hepatic IR damage. We also observed that BM-MSCs transplanted via the portal vein could attenuate IR injury by, at least in part, suppressing the oxidative stress response and inhibiting apoptosis.

\section{Acknowledgements}

The authors thank Dr Liang Hongsheng and Dr Du Wenzhong for their technical assistance.

\section{References}

1. Carden DL and Granger DN: Pathophysiology of ischaemiareperfusion injury. J Pathol 190: 255-266, 2000.

2. Nieuwenhuijs VB, De Bruijn MT, Padbury RT and Barritt GJ: Hepatic ischemia-reperfusion injury: roles of $\mathrm{Ca}^{2+}$ and other intracellular mediators of impaired bile flow and hepatocyte damage. Dig Dis Sci 51: 1087-1102, 2006.

3. Jaeschke H, Smith CV and Mitchell JR: Hypoxic damage generates reactive oxygen species in isolated perfused rat liver. Biochem Biophys Res Commun 150: 568-574, 1988.

4. Jaeschke $\mathrm{H}$ and Mitchell JR: Mitochondria and xanthine oxidase both generate reactive oxygen species in isolated perfused rat liver after hypoxic injury. Biochem Biophys Res Commun 160 140-147, 1989.

5. Jaeschke H, Bautista AP, Spolarics Z and Spitzer JJ: Superoxide generation by Kupffer cells and priming of neutrophils during reperfusion after hepatic ischemia. Free Radic Res Commun 15: 277-284, 1991

6. Shibuya H, Ohkohchi N, Seya K and Satomi S: Kupffer cells generate superoxide anions and modulate reperfusion injury in rat livers after cold preservation. Hepatology 25: 356-360, 1997.

7. Walsh KB, Toledo AH, Rivera-Chavez FA, Lopez-Neblina F and Toledo-Pereyra LH: Inflammatory mediators of liver ischemiareperfusion injury. Exp Clin Transplant 7: 78-93, 2009.

8. Arumugam TV, Magnus T, Woodruff TM, Proctor LM, Shiels IA and Taylor SM: Complement mediators in ischemia-reperfusion injury. Clin Chim Acta 374: 33-45, 2006.

9. Chang WJ and Toledo-Pereyra LH: Toll-like receptor signaling in liver ischemia and reperfusion. J Invest Surg 25: 271-277, 2012.

10. Caplan AI and Dennis JE: Mesenchymal stem cells as trophic mediators. J Cell Biochem 98: 1076-1084, 2006.

11. Borlongan CV, Glover LE, Tajiri N, Kaneko Y and Freeman TB: The great migration of bone marrow-derived stem cells toward the ischemic brain: therapeutic implications for stroke and other neurological disorders. Prog Neurobiol 95: 213-228, 2011.

12. Li SC, Acevedo J, Wang L, et al: Mechanisms for progenitor cellmediated repair for ischemic heart injury. Curr Stem Cell Res Ther 7: 2-14, 2011.

13. Wise AF and Ricardo SD: Mesenchymal stem cells in kidney inflammation and repair. Nephrology 17: 1-10, 2011.

14. Pan RL, Wang P, Xiang LX and Shao JZ: Delta-like 1 serves as a new target and contributor to liver fibrosis down-regulated by mesenchymal stem cell transplantation. J Biol Chem 286: 12340-12348, 2011.

15. Kuo TK, Hung SP, Chuang CH, et al: Stem cell therapy for liver disease: parameters governing the success of using bone marrow mesenchymal stem cells. Gastroenterology 134: 2111-2121, 2008.

16. Banas A, Teratani T, Yamamoto Y, et al: IFATS collection: in vivo therapeutic potential of human adipose tissue mesenchymal stem cells after transplantation into mice with liver injury. Stem Cells 26: 2705-2712, 2008.
17. Zhang XM, Du F, Yang D, et al: Transplanted bone marrow stem cells relocate to infarct penumbra and co-express endogenous proliferative and immature neuronal markers in a mouse model of ischemic cerebral stroke. BMC Neurosci 11: 138, 2010.

18. Nauta RJ, Tsimoyiannis E, Uribe M, Walsh DB, Miller D and Butterfield A: Oxygen-derived free radicals in hepatic ischemia and reperfusion injury in the rat. Surg Gynecol Obstet 171: $120-125,1990$

19. Suzuki S, Nakamura S, Koizumi T, et al: The beneficial effect of a prostaglandin I2 analog on ischemic rat liver. Transplantation 52: 979-983, 1991.

20. Xue T, Luo P, Zhu H, et al: Oxidative stress is involved in Dasatinib-induced apoptosis in rat primary hepatocytes. Toxicol Appl Pharmacol 261: 280-291, 2012.

21. Gavrieli Y, Sherman Y and Ben-Sasson SA: Identification of programmed cell death in situ via specific labeling of nuclear DNA fragmentation. J Cell Biol 119: 493-501, 1992.

22. Li SC, Wang L, Jiang H, Acevedo J, Chang AC and Loudon WG: Stem cell engineering for treatment of heart diseases: potentials and challenges. Cell Biol Int 33: 255-267, 2009.

23. Petit I, Jin D and Rafii S: The SDF-1-CXCR4 signaling pathway: a molecular hub modulating neo-angiogenesis. Trends Immunol 28: 299-307, 2007.

24. Chen L, Tredget EE, Wu PY and Wu Y: Paracrine factors of mesenchymal stem cells recruit macrophages and endothelial lineage cells and enhance wound healing. PLoS One 3: e1886, 2008.

25. Jin SZ, Meng XW, Sun X, et al: Hepatocyte growth factor promotes liver regeneration induced by transfusion of bone marrow mononuclear cells in a murine acute liver failure model. J Hepatobiliary Pancreat Sci 18: 397-405, 2011.

26. McCord JM: Oxygen-derived free radicals in postischemic tissue injury. N Engl J Med 312: 159-163, 1985.

27. Zhang D, Jiang M and Miao D: Transplanted human amniotic membrane-derived mesenchymal stem cells ameliorate carbon tetrachloride-induced liver cirrhosis in mouse. PLoS One 6: e16789, 2011.

28. Cho KA, Woo SY, Seoh JY, Han HS and Ryu KH: Mesenchymal stem cells restore $\mathrm{CCl}_{4}$-induced liver injury by an antioxidative process. Cell Biol Int 36: 1267-1274, 2012.

29. Chen YT, Sun CK, Lin YC, et al: Adipose-derived mesenchymal stem cell protects kidneys against ischemia-reperfusion injury through suppressing oxidative stress and inflammatory reaction. J Transl Med 9: 51, 2011.

30. Guzun R, Timohhina N, Tepp K, et al: Regulation of respiration controlled by mitochondrial creatine kinase in permeabilized cardiac cells in situ. Importance of system level properties. Biochim Biophys Acta 1787: 1089-1105, 2009.

31. Wang GW, Klein JB and Kang YJ: Metallothionein inhibits doxorubicin-induced mitochondrial cytochrome c release and caspase-3 activation in cardiomyocytes. J Pharmacol Exp Ther 298: 461-468, 2001.

32. Waetzig GH, Rosenstiel P, Arlt A, et al: Soluble tumor necrosis factor (TNF) receptor-1 induces apoptosis via reverse TNF signaling and autocrine transforming growth factor-beta1. FASEB J 19: 91-93, 2005

33. Bueno OF, De Windt LJ, Tymitz KM, et al: The MEK1-ERK1/2 signaling pathway promotes compensated cardiac hypertrophy in transgenic mice. EMBO J 19: 6341-6350, 2000.

34. Coriat R, Leconte M, Kavian N, et al: Mangafodipir protects against hepatic ischemia-reperfusion injury in mice. PLoS One 6: e27005, 2011.

35. Yu HC, Qin HY, He F, et al: Canonical notch pathway protects hepatocytes from ischemia/reperfusion injury in mice by repressing reactive oxygen species production through JAK2/ STAT3 signaling. Hepatology 54: 979-988, 2011.

36. Angoulvant D, Ivanes F, Ferrera R, Matthews PG, Nataf S and Ovize M: Mesenchymal stem cell conditioned media attenuates in vitro and ex vivo myocardial reperfusion injury. J Heart Lung Transplant 30: 95-102, 2010.

37. Shroff EH, Snyder CM, Budinger GR, et al: $\mathrm{BH} 3$ peptides induce mitochondrial fission and cell death independent of BAX/BAK. PLoS One 4: e5646, 2009.

38. Liu L, Cao JX, Sun B, et al: Mesenchymal stem cells inhibition of chronic ethanol-induced oxidative damage via upregulation of phosphatidylinositol-3-kinase/Akt and modulation of extracellular signal-regulated kinase $1 / 2$ activation in PC12 cells and neurons. Neuroscience 167: 1115-1124, 2010.

39. Zhuang S and Schnellmann RG: A death-promoting role for extracellular signal-regulated kinase. J Pharmacol Exp Ther 319: 991-997, 2006. 